\title{
Measures of skin conductance and heart rate in alcoholic men and women during memory performance
}

Kayle S Sawyer, Alan Poey, Susan Mosher Ruiz, Ksenija Marinkovic, Marlene Oscar-Berman

We examined abnormalities in physiological responses to emotional stimuli associated with long-term chronic alcoholism. Skin conductance responses (SCR) and heart rate (HR) responses were measured in 32 abstinent alcoholic (ALC) and 30 healthy nonalcoholic (NC) men and women undergoing an emotional memory task in an MRI scanner. The task required participants to remember the identity of two emotionally-valenced faces presented at the onset of each trial during functional magnetic resonance imaging (fMRI) scanning. After viewing the faces, participants saw a distractor image (an alcoholic beverage, nonalcoholic beverage, or scrambled image) followed by a single probe face. The task was to decide whether the probe face matched one of the two encoded faces. Skin conductance measurements (before and after the encoded faces, distractor, and probe) were obtained from electrodes on the index and middle fingers on the left hand. HR measurements (beats per minute before and after the encoded faces, distractor, and probe) were obtained by a pulse oximeter placed on the little finger on the left hand. We expected that, relative to NC participants, the ALC participants would show reduced SCR and $H R$ responses to the face stimuli, and that we would identify greater reactivity to the alcoholic beverage stimuli than to the distractor stimuli unrelated to alcohol. While the beverage type did not differentiate the groups, the ALC group did have reduced skin conductance and $\mathrm{HR}$ responses to elements of the task, as compared to the NC group. 
Kayle S. Sawyer ${ }^{1,2,3}$, Alan Poey ${ }^{1,2,3}$, Susan Mosher Ruiz,2,3 Ksenija Marinkovic ${ }^{4}$, and

Marlene Oscar-Berman $1,2,3$

4

$5 \quad{ }^{1}$ Boston University School of Medicine, Boston, MA; ${ }^{2 V A}$ Boston Healthcare System, Boston,

$6 \mathrm{MA} ;{ }^{3}$ Athinoula A. Martinos Center for Biomedical Imaging, Massachusetts General Hospital,

7 Boston, MA; ${ }^{4}$ University of California at San Diego, CA, and Psychology Department, San

Diego State University, CA

9 Correspondence:

10 Kayle S Sawyer, Ph.D.

11 Boston University School of Medicine, Suite L-815

1272 East Concord Street, Boston, MA 02118

13 Tel: 617-638-4803, Fax: 617-638-4806, e-mail: kslays@bu.edu

14

15 Running Head: Psychophysiological Measures in Alcoholic Men and Women

16 
Introduction

18 Abstinent alcoholics are impaired on many cognitive and emotional tasks (Oscar-Berman et

19 al., 2014). Most investigations into these phenomena have focused on deficits reflective of

20 abnormalities of the central nervous system (Makris et al., 2008) and of overt behaviors

21 (Oscar-Berman et al., 2014), with less attention devoted to dysregulation of the autonomic

22 nervous system (ANS) (Karpyak et al., 2014; Boschloo et al., 2011). Despite a certain degree

23 of autonomy of the ANS in its control over the internal physiological milieu, central and

24 autonomic components of the nervous system work in synchrony and interact in reciprocal

25 ways on numerous levels of the neuraxis, regulating behavior in a seamlessly integrated

26 manner (Clore \& Ortony, 2000; Critchley et al., 2013; Damasio, 1998). Nevertheless, their

27 functional profiles and measurement methods are distinct. Peripheral autonomic measures

28 may help discern effects of chronic alcoholism on the neurofunctional systems that comprise

29 emotional dimensions. They also may provide insight into brain modulatory processes such

30 as arousal circuits that moderate cognition, including working memory (Dolcos \& McCarthy,

31 2006). In the present study, we examined the peripheral responses of electrodermal activity

32 and heart rate (HR), two commonly used indicators of ANS reactivity, in alcoholic (ALC) and

33 nonalcoholic control (NC) individuals while they viewed emotionally evocative visual stimuli.

34 Electrodermal activity reflects skin conductivity (tonic, slow changes over time) and

35 skin conductance responses (SCRs; short-lasting phasic changes in response to discrete

36 stimuli). The conductivity of the skin is primarily influenced by the amount of moisture

37 produced by eccrine sweat glands under control of the sympathetic branch of the ANS and is

38 therefore an excellent indicator of the overall arousal state (the level of skin conductance) or

39 stimulus-induced arousal (SCR) (Boucsein, 2012; Dawson et al., 2007; Naqvi \& Bechara,

40 2006). The HR measure (i.e., the number of beats per minute) is controlled by both the 
41 sympathetic and parasympathetic branches of the ANS (Berntson et al., 2007), and it reflects

42 stress responsiveness in psychosocial and cognitive situations (Karpyak et al., 2014; Starcke

43 et al., 2013). These measures have been the methods of choice in numerous studies

44 investigating functions that rely on affective dimensions including lie detection, orienting to

45 novelty, classical conditioning, and processing of stimuli varying in emotional valence and

46 significance (Dawson et al., 2007; Maltzman, 1990; Lang et al., 1993; MacLaren, 2001), as

47 well as decision making (Naqvi \& Bechara, 2006). Furthermore, these peripheral indices

48 provide insight into psychopathological conditions such as schizophrenia (Schell et al., 2005),

49 psychopathy (Fowles, 1993), and depression (Thorell, 2009). In the case of alcoholism, these

50 indices complement behavioral or neuroimaging measures that do not address ANS

51 reactivity. Indeed, long-term alcohol abuse results in diverse abnormalities of ANS functions.

52 For example, reduced sweating is observed in chronic alcoholics diagnosed with peripheral

53 neuropathy (Low et al., 1975), and post mortem studies indicate degeneration of the

54 sympathetic and vagus nerves (Novak \& Victor, 1974).

Neuroanatomical substrates that influence electrodermal and cardiac response systems consist of distributed and interrelated limbic and cortical circuitries. Direct electrical

57 stimulation of limbic structures, primarily the amygdala, hippocampus, and anterior cingulate

58 gyrus, have been shown to evoke large SCRs, whereas stimulation of cortical areas such as

59 frontal and mid-temporal regions have been shown to result in a weaker, but still observable

60 modulation (Mangina \& Beuzeron-Mangina, 1996). Neuropsychological research has shown

61 that lesions of the ventromedial prefrontal, anterior cingulate, and right parietal areas result in

62 reduced electrodermal activity (Critchley, 2002; Tranel, 2000). Studies using simultaneous

63 measures of central (fMRI), and autonomic activity $(\mathrm{HR})$ have provided insight into the control 
64 of HR. Activity in the amygdala, insula, anterior cingulate, and brainstem predict changes in

$65 \mathrm{HR}$ in response to emotional faces (Critchley, 2005).

Levels of physiological arousal have been positively associated with level of dependence and consumption in responses to alcohol cue exposure (Sinha et al., 2009;

68 Carter \& Tiffany, 1999), and gender differences in these responses also have been reported

69 (Nesic \& Duka, 2006), although not in relation to alcoholism. Chronic alcoholism also may be

70 differently associated with HR cue reactivity. That is, ALC individuals have shown attenuated

71 HR responses to alcohol cues in a Stroop paradigm (Stormark et al., 2000), and their HR

72 responses to alcohol cues have been shown to differentiate beverage preferences (Staiger \&

73 White, 1991). However, when compiling a meta-analysis of $11 \mathrm{HR}$ and 9 SCR cue-reactivity

74 studies, Carter and Tiffany (1999) found that alcoholics did have higher reactivity to alcohol-

75 related cues than to neutral cues. Of note, the perception of alcohol-related cues involves

76 limbic structures important for emotional functions. These structures also are used in the

77 processing of emotional faces, which has been observed to be impaired in alcoholics

78 (Marinkovic et al., 2009).

The present study examined the intensity of ANS responses to two types of stimuli with

80 differing emotional characteristics (facial expressions and beverage cues), and how they

81 interacted. The stimuli were presented in the context of a memory task that was designed to

82 investigate how emotional memory for faces would be influenced by subsequent distraction

83 by alcohol cues, and whether the reactivity to those alcohol cues would be differentially

84 modulated by positive or negative facial expressions (Ruiz, 2012). In addition to examining

85 the influence of alcoholism on ANS responses, we also sought to explore if the effects we

86 observed were different for men and women, and if those gender effects differed for the ALC

87 and NC groups. In accordance with the literature described, we predicted that ALC 
88 participants would have abnormal SCR and HR responsivity to the facial and beverage cues

89 presented in our task, and that men and women would display divergent ANS patterns.

90 Specifically, we predicted that relative to NC participants, the ALC participants would have

91 lower SCR and HR responses to the face stimuli, and greater reactivity to the alcoholic

92 beverage stimuli than to the stimuli unrelated to alcohol. We also predicted that women would

93 show greater ANS responses to the faces than men, in accordance with gender differences

94 typically reported in psychophysiological responses to emotional stimuli (e.g., Bianchin \&

95 Angrilli, 2012), and we sought to explore how this gender difference would be represented in

96 ALC participants.

In both acute and chronic alcohol exposure, orienting responses can be abnormal.

98 Marinkovic and colleagues (2001) found that small amounts of alcohol (about one or two

99 drinks) in healthy controls attenuated electrical activity of the brain (as measured by the event

100 related brain potential of the orienting response) to novel sounds on trials with corresponding

101 autonomic arousal (i.e., SCR). This indicates that the brain may be less sensitive to novel or

102 rarely occurring stimuli under the influence of alcohol, and that the SCR is a concomitant

103 indicator of this orienting response. Croissant and colleagues (2006) observed that acute

104 alcohol administration attenuated the HR increases observed in response to aversive sounds

105 and reward stimuli, but the effects depended upon gender and family history of alcoholism.

106 Ray and colleagues (2006) reported that heavy drinkers with heightened HR reactivity to

107 intravenous alcohol infusion were more sensitive to the invigorating properties of alcohol,

108 while being less sensitive to the sedative and unpleasant effects of alcohol intoxication.

109 Interestingly, we showed that alcoholic Korsakoff patients (with limbic and prefrontal damage)

110 had low electrodermal orienting responses to unexpected loud sounds (Oscar-Berman \&

111 Gade, 1979). While orienting responses typically are associated with a reduction in HR 
112 (Weisbard \& Graham, 1971), in the present study we predicted that these responses would

113 be abnormal in ALC participants.

114 In summary, essential involvement of limbic and prefrontal networks in the regulation

115 and modulation of electrodermal and cardiovascular activity makes these measures valuable

116 for studying emotional functioning as affected by alcoholism. Therefore, we measured SCR

117 and HR in ALC and NC participants undergoing an emotional memory task. Because a

118 number of studies have found increased neural reactivity when ALCs are exposed to alcohol

119 cues (Wrase et al., 2002; Myrick et al., 2004), our task employed alcohol distractor cues

120 during a memory maintenance period for faces with emotional expressions. We predicted that

121 ALCs would have abnormal physiological responses to the two different emotional

122 characteristics (facial expressions and beverage cues), and that these responses would be

123 different for men and women. Finally, we sought to explore (a) how face valence and

124 distractor type would interact, and (b) how the memory probe faces would differentiate ANS

125 responsivitity for ALC men and women.

\section{Materials and Methods}

128 Participants. A total of 62 individuals from the Boston area were included in the study (see

129 Table 1). One group consisted of 32 abstinent ALC men and women, and the other group

130 consisted of 30 gender- and age-equivalent healthy NC individuals. The task in which they

131 participated took place in an MRI scanner, where we collected neuroimaging and behavioral

132 data concurrently with psychophysiological measures. (The neuroimaging and behavioral

133 results are described separately by Ruiz, 2012.) Originally, SCR and HR data were collected

134 from 76 participants. However, because of problems encountered during physiological

135 recording in the MRI scanner (e.g., MRI radio frequency interference, motion induced artifacts 
136 from the static magnetic field, poor skin responsivity with electrodes, other motion artifacts,

137 etc.), we had good quality data with low levels of noise and easily discernable SCRs for 62

138 participants. From these 62 participants, data for a total of 46 individuals were available for

139 analyses of each of the two physiological measures (see Supplemental Tables 1 and 2 for the

140 distribution of participants into SCR or HR subgroups, and see Supplemental Raw

141 Physiological Data: figshare, http://dx.doi.org/10.6084/m9.figshare.1025792). Participants

142 responded to flyers posted online, at the VA Boston Healthcare System and the Boston

143 University Medical Center, and in public places (churches, stores, etc.). This research was

144 approved by the Institutional Review Boards of Boston University School of Medicine

145 (\#H24686), VA Boston Healthcare System (\#1017 and \#1018), and Massachusetts General

146 Hospital (\#2000P001891). Participants were reimbursed for time and travel expenses.

Selection procedures included an initial structured telephone interview to determine

148 age, level of education, health history, and history of alcohol and drug use. To be brought in

149 for neuropsychological testing, electrophysiological measurements, and neuroimaging, all

150 potential participants were required to be right-handed, have normal or corrected-to-normal

151 vision, and speak English as their first language (or have acquired English as a second

152 language by five years of age). Eligible individuals were invited to the laboratory for further

153 screening and evaluations.

154 Participants received a structured interview regarding their drinking patterns, including

155 length of abstinence and duration of heavy drinking (DHD), i.e., the number of years they

156 consumed more than 21 drinks per week (one drink: $355 \mathrm{~mL}$ beer, $148 \mathrm{~mL}$ wine, or $44 \mathrm{~mL}$

157 hard liquor). A Quantity Frequency Index (QFI; Cahalan et al., 1969), which roughly

158 corresponds to number of daily drinks (at one ounce of ethanol per drink), was calculated for

159 each participant. This measure evaluates the amount, type, and frequency of alcohol usage 
160 over the last six months (for the NC participants), or over the six months preceding cessation

161 of drinking (for the ALC participants). The ALC participants met DSM-IV criteria for moderate

162 to severe lifetime alcohol abuse or dependence, and had a minimum duration of five years of

163 heavy drinking (at 21 or more drinks per week). All ALCs had abstained from drinking alcohol

164 for at least four weeks prior to testing (except one ALC man who had a drink three days prior

165 to the study and had ceased heavy drinking eight years earlier).

166 Neurobehavioral and psychiatric evaluations typically required six to nine hours over

167 three or more days. Participants had frequent breaks, and sessions were discontinued and

168 rescheduled if a participant indicated fatigue. Participants underwent a medical history

169 interview and vision testing, plus a series of questionnaires (e.g., handedness, alcohol and

170 drug use) to ensure they met inclusion criteria. In order to minimize confounding effects from

171 illicit drug use, psychoactive drug use, and psychiatric comorbidity, participants were given an

172 extensive battery of screening tests. They performed a computer-assisted, shortened version

173 of the Diagnostic Interview Schedule (DIS; Robins et al., 2000) Version IV that provides

174 lifetime psychiatric diagnoses according to the Diagnostic and Statistical Manual criteria

175 (DSM-IV-TR; APA, 2000). Individuals were excluded from further participation if any source

176 (DIS scores, hospital records, referrals, or personal interviews) indicated that they had any of

177 the following: Korsakoff's syndrome; HIV; hepatitis; cirrhosis; major head injury with loss of

178 consciousness greater than 20 minutes; stroke; epilepsy or seizures unrelated to alcoholism;

179 Hamilton Rating Scale for Depression (Hamilton, 1960) score over 14; major depressive

180 disorder; bipolar I or II disorder; schizoaffective disorder; schizophreniform disorder;

181 schizophrenia; generalized anxiety disorder; or electroconvulsive therapy. Additionally, we

182 excluded individuals who failed screening for MRI safety (e.g., metal implants, obesity,

183 pregnancy). 
184 Neuropsychological Assessment. In order to collect demographic information, all

185 participants were given a battery of neuropsychological tests, and the following IQ and

186 memory measures (Wechsler, 1997) were examined: The Wechsler Adult Intelligence Scale

187 (WAIS-III) Verbal, Performance, and Full Scale Intelligence Quotient scores (VIQ, PIQ, and

$188 \mathrm{FSIQ}$ ) and the Wechsler Memory Scale (WMS) Immediate, Delayed, and Working Memory

189 scores. Independent-samples t-tests did not reveal significant differences among the ALC and

190 NC groups for the WAIS and WMS measures.

191 Memory Task. All participants were given a delayed memory task in the MRI scanner, in

192 which face and alcoholic beverage stimuli were displayed. The task was designed to assess

193 multiple cognitive functions. The primary goals were to assess ANS reactivity (SCR and HR)

194 to emotional face stimuli and to alcohol cues. The secondary goal of the task was to examine

195 the interaction of these factors (Face Emotion $x$ Distractor Type) in order to characterize its

196 influence during the distractor element and the memory probe (not on memory performance

197 itself, which is addressed elsewhere; Ruiz, 2012).

198 Our task has been described in detail by Ruiz (2012). Figure 1 shows the flow of the

199 task for three example trials. On each trial, pictures of two faces were displayed

200 simultaneously for three seconds, followed by a fixation asterisk $\left(^{*}\right)$ for one second (the

201 encoded faces). The participants were asked to maintain these faces in memory while a

202 colored distractor stimulus was shown (the distractor element). Each distractor picture was

203 shown for three seconds, followed by a fixation asterisk $\left({ }^{*}\right)$ for one second. Following the

204 distractor picture, a single probe face was shown for two seconds (the probe element), and

205 the participants were required to report whether this face was one of the two faces they had

206 just seen. Each trial was 10 seconds in length, and was followed by a variable delay period

207 (with a mean duration of 10 seconds, ranging from 2-22 seconds) during which the 
208 participants engaged in fixation on a set of crosshairs (+++). The delay period was designed

209 to be of sufficient duration to assess orienting to the encoded faces that followed it, for which

210 we predicted abnormal ANS responses in the ALC group.

211 The face stimuli were divided into three valence types by facial expression: positive,

212 negative, and neutral (the Face Valence). Photographs were of unfamiliar adults without

213 glasses, facial hair, or jewelry. The faces were shown in grayscale against a black

214 background. The faces were balanced to contain $50 \%$ male and $50 \%$ female faces. The

215 photographs were evaluated for the consistency of emotional expressions by a group of

216 independent judges, and we selected their most consistent choices (Marinkovic \& Halgren,

217 1998). We predicted that because the brain structures responsible for ANS responses to

218 emotional faces are dysregulated in alcoholics, the ALC group would have attenuated ANS

219 responses to the emotional faces.

220 On different trials, the distractor stimulus was either a picture of an alcoholic beverage

221 (beer, wine, liquor, or mixed drink), a picture of a nonalcoholic beverage (water, juice, milk,

222 soda, coffee, tea, etc.), or a scrambled nonsense picture constructed to have similar colors

223 and brightness as the other distractors (the Distractor Type). Alcoholic and nonalcoholic

224 beverage pictures were a combination of images used with permission from the Normative

225 Appetitive Picture System (NAPS) (Stritzke et al., 2004), and other previously published

226 works on alcohol cues (Wrase et al., 2002; Myrick et al., 2004). Additional distractor images

227 were modified from digital photographs taken at bars, liquor stores, and convenience stores.

228 The scrambled images were created by inverting half the alcoholic and half the nonalcoholic

229 beverage images and distorting them until they were not recognizable as objects. In

230 accordance with our hypotheses, we expected that the ALC group would have stronger

231 neuropsychological responses to the alcohol cues than the nonalcohol cues, and that this 
232 difference would be reflected by abnormal ANS responses. Further, we predicted this

233 difference would be more pronounced for the ALC group than the NC group.

234 The probe face matched one of the encoded faces on $50 \%$ of the trials, and

$235 \mathrm{match} / \mathrm{mismatch}$ trials appeared in a randomized order within each run (the Probe Match).

236 Responses were made by pressing one of two buttons with the index finger (match) or middle

237 finger (mismatch) of the right hand. Participants were instructed to respond as quickly as

238 possible without sacrificing accuracy. Additionally, they were told that if they felt their first

239 response was incorrect, they could immediately correct the response by pressing the

240 opposite button as soon as possible after the error was detected.

241 The task was divided into nine runs, each of which contained 18 trials. There were nine

242 trial types made up of each combination of Face Valence, Distractor Type (e.g., positive faces

243 followed by alcohol distractor), and Probe Match status, for a total of 18 trial types. Each

244 emotion-distractor combination appeared twice per run. In total, there were 54 trials for each

245 type of distractor (combined across Face Valence) and each face emotion (combined across

246 Distractor Type), for a total of 162 trials across the entire scan. A total of 162 different faces

247 (balanced by gender) were used in the study. Within a trial, the two encoded faces and the

248 probe face had the same emotional expression and gender. This way, on match trials the

249 probe facial image was identical to one of the encoded images, and on mismatch trials the

250 facial identity changed but the emotional expression and gender did not.

251 An IBM ThinkPad running Presentation version 11.2 for Windows XP (NeuroBehavioral

252 Systems, Albany, CA) software was used for visual presentation of the experimental stimuli

253 and collection of participants' responses in the scanner. Equipment for the task consisted of a

254 shielded projector and screen, mirrors, and button box. Stimuli were back-projected onto a 
255 screen at the back of the scanner bore and were viewed by the participants through a mirror

256 mounted to the head coil. All participants wore earplugs to attenuate scanner noise.

257 Psychophysiological Measures. Psychophysiological data for skin conductance and pulse

258 oximetry (for HR) were collected with MRI-compatible equipment simultaneously with fMRI

259 data collection during the memory task. Tonic levels and phasic changes of skin conductance

260 were recorded from MLT117F silver-silver chloride contoured finger electrodes filled with

261 BioPac GEL101 isotonic electrode gel. Electrodes were attached to the index and middle

262 fingers of the left hand with velcro straps, leaving the right hand free for performing the task.

263 Skin conductance was amplified using an ADInstruments PowerLab 16SP with ML116 GSR

264 Amplifier, and recorded digitally using ADInstruments LabChart software. It was recorded

265 continuously throughout the functional scans, which allowed us to gather electrodermal

266 responses elicited during the task. Preprocessing included attenuation of noise elements via

267 individualized notch filters for signal induced from respiratory and pulsatile movements within

268 the magnetic field by targeting the frequencies obtained from simultaneous etCO2

269 measurement (approximately $0.3 \mathrm{~Hz}$ ) and from pulse oximetry determined $\mathrm{HR}$ (at

270 approximately $1.2 \mathrm{~Hz}$ ). Fourier transformed power spectra were examined for other sources

271 of noise, and further notch filters were applied as needed.

272 HR was obtained through a pulse oximeter attached to the little finger on the left hand.

273 HR was amplified by an Invivo Magnitude 3155A system, relayed to the ADInstruments

274 PowerLab. LabChart software was utilized to identify the peak slope, and pulse identification

275 was manually examined across each run for each subject. HR was calculated using the inter-

276 beat interval and smoothed with a one second window. To account for signal dropouts, a 50

277 second moving window average was calculated, and any inter-beat intervals deviating by 
278 more than 25 BMP were replaced with the moving window average. We did not correct for 279 respiratory sinus arrhythmia.

280 Statistical Analyses. SPSS version 20 (Armonk, NY: IBM Corp) was used for statistical 281 analyses, and Excel 2011 was used for graphing (Redmond, WA: Microsoft). For each 282 element of the memory task (encoded faces, distractor element, and probe element), skin 283 conductance was analyzed for amplitude increases. Specifically, the SCR amplitude obtained 284 for each stimulus was calculated by measuring the level of increase from (a) the mean 285 conductance level obtained during the three seconds preceding the element, to (b) the peak 286 conductance during the six seconds following the element. In other words, the SCR for each 287 trial was defined as the amount of conductance increase for that element (Dawson et al., 288 2007). This was accomplished automatically with LabChart software, without the experimenters' input. Then, in an effort to minimize the effects of occasional outliers that were due to movement or other types of artifacts, we detected individual trial SCRs that were greater than four standard deviations above the mean (approximately $1 \%$ of the trials), and removed them from the analyses.

We assessed the skin conductance responsivity in two ways that allowed us to obtain

294 the average, i.e., representative, values for the factors of interest without individual trial 295 variability. First, in order to get insight into the overall level of responsivity, we obtained the 296 counts of responses that were greater than $0.03 \mu S$ irrespective of their amplitude.

297 Additionally, we analyzed response amplitudes by averaging them across all runs for each 298 trial type irrespective of the response frequency. Models were constructed for the responses 299 to the encoded faces, distractor element, and probe element separately. Between-subjects 300 interaction effects were included to test the influence of preceding stimuli. The data were 301 analyzed with full-factorial mixed-design analyses of variance (ANOVA) that included: (a) 
302 between-group factors of Group and Gender, and (b) the relevant within-subject factors of

303 Face Valence, Distractor Type, and Probe Match, and all interactions of these factors

304 (Woodward, Bonett, \& Brecht, 1990).

As with skin conductance analyses, HR changes were examined for each element of

306 the task. Difference values were obtained by subtracting the mean HR in the two seconds

307 following the element from the two seconds preceding it. As with the SCR analyses, outliers

308 were detected and removed, defined as any single-trial HR changes greater than five beats

309 per minute (BPM) or exceeding four standard deviations. Around $1 \%$ of trials were removed in

310 this manner. As with the SCR analyses, the HR data were averaged across the stimulus

311 categories included in each ANOVA design, which used Group and Gender as between-

312 group factors, and Face Valence, Distractor Type, and Probe Match as within-subject factors,

313 along with all interactions of these factors.

314 For both SCR and HR analyses, the full-factorial mixed-design ANOVA models

315 simultaneously provided results not only for the specific predictions to our hypotheses, but

316 also for further exploratory analyses. That is, the ANOVA models for the encoded faces

317 tested our predictions that the orienting responses would be reduced for the ALC group, and

318 that the responses to the emotional faces would be diminished, while additionally including

319 other exploratory effects specified in the full-factorial model. For the distractor element, the

320 ANOVA models included factors to examine our prediction that the alcohol cues would elicit

321 stronger ANS responses for the ALC group, and the Group x Distractor Type interaction

322 would investigate whether this difference were more pronounced for the ALC group than for

323 the NC group. Further analyses, including an examination of how the face valence differently

324 influenced responses to each distractor type (Face Valence $x$ Distractor Type interaction),

325 were conducted on an exploratory basis. Interactions with Gender were included in the 
326 models to address our hypothesis that ANS reactions would be stronger for the women than

327 for the men, and to explore how this difference is represented among alcoholics.

329 Results

330 The results of the SCR and HR measures were in line with our predictions that relative to NC

331 participants, the ALC participants would show reduced SCR and HR responses to the face

332 stimuli. We did not find evidence to support our hypotheses that alcoholics would have

333 greater reactivity to the alcoholic beverage stimuli than to the distractor stimuli unrelated to

334 alcohol, nor that women would demonstrate greater ANS reactivity than men.

335 Skin Conductance. Both types of skin conductance measures, i.e., response counts and

336 response amplitudes, were analyzed for each of the task elements (encoded faces, distractor

337 element, and probe element). In concert with our predictions, ALC participants manifested

338 lower skin conductance responsivity than the NCs, and this was particularly apparent for the

339 ALC women. In addition to Group and Gender, SCR was sensitive to emotional face

340 expressions and the matching status of the probe element. Results of the analyses for each

341 of the three task elements are presented seriatim.

Encoded faces. A mixed-design ANOVA was used that included the following factors:

343 Group, Gender, and Face Valence, averaging across the other conditions before statistical

344 analyses. For the SCR counts, the significant main effect of Group $(F(1,41)=7.9, p<0.01)$

345 indicated that the ALC participants produced 10 percentage points fewer responses above

$3460.03 \mu \mathrm{S}$ than the NCs, as predicted. This effect was particularly evident for female

347 participants $(F(1,41)=5.5, p<0.05$; Figure 2), although the Group $\times$ Gender interaction was

348 not significant $(F(1,41)=0.9, p=0.34)$. Analyses of the SCR amplitudes did not reveal

349 significant results for our hypotheses that the encoded faces would elicit a diminished 
350 orienting response in alcoholics $(\mathrm{F}(1,41)=1.8, p=0.18)$, nor abnormal responses to the

351 emotional faces as indicated by a Group x Face Valence interaction $(F(2,82)=0.5, p=0.61)$.

352 Exploratory analyses did not yield significant results.

353 Distractor element. A mixed-design ANOVA was used that included the following

354 factors: Group, Gender, Face Valence, and Distractor Type, averaging across the other

355 conditions before the analyses. As was the case for encoded faces, the ALC participants

356 were significantly less responsive than the NCs to the distractors, as reflected in 13

357 percentage points fewer measurable SCRs $(F(1,41)=11.3, p<0.01)$. The ALC women were

358 significantly less responsive than the NC women $(F(1,41)=8.2, p<0.001)$. A more detailed,

359 but consistent picture emerged when the SCR amplitudes were analyzed (Figure 3), although

360 the predicted Group $\times$ Distractor Type interaction was not identified $(F(2,82)=1.1, p=0.34$;

361 Figure 4). The main effect of Group $(F(1,41)=2.8, p=0.1)$ was especially evident for the

362 difference between the female ALCs and NCs $(F(1,41)=4.2, p<0.05)$, though the

363 interaction was not significant $(F(1,41)=1.7, p=0.20)$, nor was the exploratory analysis of

364 Face Valence $x$ Distractor Type $(F(4,164)=0.9, p=0.45)$.

Probe element. A mixed-design ANOVA included the following factors: Group,

366 Gender, Face Valence, Distractor Type, and Probe Match (averaging across runs), and was

367 applied to explore effects for both types of skin conductance measures. The significant main

368 effect of Group $(F(1,41)=6.6, p<0.05)$ for the SCR counts once again confirmed that the

369 ALC group produced 14 percentage points fewer SCRs than the NC group, and this effect

370 was particularly prominent for female participants $(F(1,41)=4.1, p<0.05)$. The main effect of

371 Probe Match $(F(1,41)=8.7, p<0.01)$, was a result of greater numbers of responses to

372 matching probe stimuli. A significant Gender $x$ Probe Match interaction $(F(1,41)=6.0, p<$ 
$3730.05)$ indicated that male participants were especially sensitive to the match/mismatch

374 difference $(F(1,41)=15.3, p<0.01$; Figure 5$)$.

375 Analyses of SCR amplitudes imparted complementary information. A significant Face

376 Valence $x$ Probe Match interaction $(F(1,41)=4.9, p<0.01)$ provided insight into the relative

377 influence of the Probe Match status vs. Face Valence. Faces with negative emotional

378 expressions elicited larger SCRs than both positive and neutral faces $(F(1,41)=5.5, p<$

$3790.05)$, confirming the sensitivity of this measure to emotionally arousing stimuli. The match

380 and nonmatch conditions did not differ significantly for negative face expressions. In contrast,

381 matching face stimuli elicited larger SCRs when the expressions were neutral $(F(1,41)=$

$38211.0, p<0.01)$ and positive $(F(1,41)=9.3, p<0.01)$. The exploratory analysis of the Face

383 Valence $\times$ Distractor Type interaction did not yield significant results $(F(4,164)=0.2, p=$

$3840.92)$.

385 Heart Rate. Differences in the HR change (see Figures 6, 7, 8, and 9) were analyzed with 386 mixed-design ANOVAs, as were performed for SCR. While our predictions of reduced ANS

387 reactions of the ALC group were generally born out, the measures of HR change did not

388 appear to be as sensitive as SCR to Group and Gender, nor to task manipulations. Results of

389 the analyses for each of the three task elements - encoded faces, distractor element, and

390 probe element — are presented seriatim.

Encoded faces. As predicted by our hypotheses, the HR response for the ALC group

392 was lower in comparison to the NC group $(F(1,39)=9.6, p<0.01$; Figure 6$)$. The Group

393 difference observed in relation to the faces was most evident among the women: The HR of

394 the ALC women was 0.3 BPM slower than the HR of the NC women $(F(1,39)=10.4, p<$

395 0.01). The difference between ALC and NC men (0.1 BPM), while in the same direction as for 
396 the women, was not significant. We did not identify a significant Group x Face Valence

397 interaction $(F(2,78)=0.01, p=0.98)$.

398 Distractor element. Analyses of the HRs of all participants combined indicated a 0.3

399 BPM overall increase in HR to the distractor element $(\mathrm{t}(42)=4.2, p<0.01)$. Figure 7 shows

400 the results of the three-way interaction of Group x Gender x Face Valence, which approached

401 significance $(F(2,78)=2.4, p=0.1)$ as indicated by larger HR increases for ALC women to

402 distractor cues following negative emotional expressions than HR increases for NC women

403 for the same condition $(F(1,39)=3.4, p<0.1)$. Our hypothesis that differential HR responses

404 to the Distractor Type would differ by Group was not confirmed $(F(2,78)=0.72, p=0.49$;

405 Figure 8).

406 Probe element. Exploratory analyses of the HRs of all participants together to the 407 probe face element revealed a significant overall HR reduction of 0.5 BPM $(\mathrm{t}(42)=-5.5, p<$

408 0.01; Figure 9). Differences in HR response also were examined for the factors of Group,

409 Gender, Face Valence, Distractor Type, and Probe Match, averaging across runs. The

410 reduction in HR was similar for the ALC and NC groups for both men (95\% confidence

411 interval of NC minus ALC: $[-0.8,0.3]$ BPM) and women ([-0.8, 0.3] BPM); no significant effects

412 relating to task conditions or interactions with Gender were observed for the probe element.

\section{Discussion}

416 In the present study, the pattern of autonomic responses to all three elements of the task

417 (encoded faces, distractor, and probe) differed for the ALC and NC groups. The electrodermal

418 measures indicated that, compared to the NC group, the ALC group evidenced fewer SCRs

419 to each of the three task elements (Figures 2 and 3), in line with our primary hypothesis. HR 
420 responses also differed between ALC and NC groups to the task elements, most notably in 421 relation to the encoded faces, wherein the ALC group had a greater HR decline than the NC

422 group (Figure 6). The ALCs had a greater HR increase than NCs to the distractor element

423 (Figure 7), but perhaps a more similar HR decrease in response to the probe element (Figure

424 9). We did not find evidence for our secondary prediction that the ALC group would have a

425 greater difference in SCR and HR response to the alcoholic beverage distractors than the

426 nonalcoholic beverage distractors (Figures 4 and 8). In our exploration of gender, we

427 determined that the alcoholism-related SCR and HR response abnormalities were

428 pronounced among women. Taken together, these abnormalities resulting from peripheral

429 ANS measures can be interpreted within the context of the central nervous system.

430 Brain systems and ANS activity. Measures of electrodermal activity and HR reflect activity

431 of the ANS, and they have been used in studies of emotional arousal, classical conditioning,

432 orienting to novelty and attention, cognitive load, and reward significance (Boucsein, 2012;

433 Andreassi, 2007; Critchley et al., 2005; Dawson et al., 2007). Autonomic responses have

434 been measured in studies of chronic alcoholics with mixed results (Maltzman \& Marinkovic,

435 1996). Some studies failed to find abnormalities in responsivity (Wrase et al., 2002; Reid,

436 2006; Pomerleau, 1983), or have observed lower sensitivity and greater habituation in

437 alcoholic participants compared to nonalcoholic controls (Finn, 2001). In the present study,

438 SCR results indicated lower sympathetic arousal levels in alcoholics, in agreement with

439 previous reports of lower skin conductance orienting responses to novel stimuli in alcoholic

440 Korsakoff patients (Oscar-Berman \& Gade, 1979). Even though the participants in the present

441 study did not exhibit Korsakoff symptomatology, the lower autonomic responsivity may be

442 indicative of mesocorticolimbic impairments, as (1) lesions of the amygdala (Bechara et al.,

443 1999) and prefrontal cortex (Bechara et al., 2005b) have been shown to result in a decrease 
444 or absence of SCRs, and (2) SCRs can be elicited by electrical stimulation of amygdala, 445 hippocampus, anterior cingulate, and frontal cortex (Mangina \& Beuzeron-Mangina, 1996). 446 Additionally, we have found (Marinkovic et al., 2009) that abstinent alcoholics, while 447 viewing faces with emotional expressions, exhibited blunted fMRI activation in the amygdala 448 and hippocampus, whereas nonalcoholic controls showed robust activation in these brain 449 areas. Moreover, the alcoholics in that study recruited the prefrontal cortex to process facial 450 emotions, possibly compensating for the limbic deficiency (Oscar-Berman et al., 2014). While

451 higher order cortical association areas allow us to understand the significance of emotional 452 situations, lower level structures, e.g., those with limbic system connections, are needed to 453 express emotions through changes in the periphery. Frontal brain systems participate in both 454 of these processes (reviewed by Damasio, 1998; Davidson et al., 2000; Hariri et al., 2003; 455 Roy et al., 2012; Stuss \& Knight, 2012), and patients with frontal system damage not only 456 lack emotional propriety, they also do not show changes in peripheral responses that 457 normally accompany emotional arousal (Bechara et al., 2005b; Gainotti, 2000).

458 Encoding of emotional faces. Our results indicated lower skin conductance reactivity for the 459 ALC group than for the NC group in response to the encoded faces, as predicted by our 460 hypotheses. This finding is consistent with reports of impaired memory for emotional facial 461 expressions (Foisy et al, 2007) and reduced cortico-limbic system reactivity to emotional 462 faces (Marinkovic et al., 2009; O’Daly et al., 2012; Salloum et al., 2007) observed in 463 association with alcoholism. The specific brain regions showing blunted activation in 464 alcoholics are the amygdala and hippocampus, as well as cingulate, orbitofrontal, and insular 465 cortex, all regions that mediate emotional processing. We further identified that HR fell more for the ALC group than the NC group in 467 response to the encoded faces. Although the literature on HR in alcoholics is limited by 
468 numerous methodological differences among studies, especially with respect to subject 469 samples, a review of 10 studies examining HR variability in alcoholics (Karpyak et al., 2014) 470 showed several consistent findings: There was a decrease in HR variability indices among 471 alcoholics compared to controls, possibly reflecting decreased parasympathetic influences, 472 with the effect size being proportional to amount of alcohol use, and improvement (more 473 variability) noted after prolonged abstinence. We expected an HR decrease for both groups to 474 the encoded faces, because a reduction is associated with a normal, healthy orienting to a 475 stimulus (Weisbard \& Graham, 1971). Unexpectedly, we observed a larger HR decrease in 476 the ALCs, which may suggest that the emotional or psychosocial content represented by the 477 faces was more arousing for the ALCs. Alternatively, it is possible that the apparently 478 opposite abnormalities observed for HR and SCR reflect the differential contributions of the 479 sympathetic and parasympathetic ANS. That is, skin conductance is influenced primarily by 480 the sympathetic ANS, while HR is controlled by both the sympathetic and parasympathetic 481 ANS (Berntson et al., 2007). Thus, the combined HR and SCR results might be related to 482 differential perturbations of the sympathetic and parasympathetic ANS in alcoholism.

483 Distractor element. Although the ALCs showed reduced SCRs to the distractors as a whole, 484 there was not evidence for differential effects that were specific to the alcohol cue distractor 485 images. While studies that have measured skin conductance in alcoholics viewing alcohol486 related stimuli typically have reported increased psychophysiological reactivity (Cooney et al., 487 1997; Laberg et al., 1992; Szegedi et al., 2000), some did not identify differences between 488 electrodermal responsivity of alcoholics and of controls (Grüsser et al., 2004; Wrase et al., 489 2002). In part, our results are consistent with elements of all these studies in that they are 490 variable and indicate small SCR effects that are not easily distinguished. These results might 491 suggest that the ALCs in this study, who had been abstinent for a mean duration of about 
492 nine years, may have successfully maintained their sobriety in part because they no longer

493 experience as intense ANS responses specific to alcohol-related stimuli. While we did detect

494 mildly muted SCR to distractor cues in ALC participants, we did not identify as clear a pattern

495 related to HR responses.

496 Our task was designed with the secondary aim to examine how the preceding

497 emotional valence of the faces would influence ANS responses to the distractor element.

498 However, we did not identify a significant interaction, likely due to the relatively low sensitivity

499 of these autonomic measures, and by the fact that the faces were not very effective in eliciting

500 emotional arousal.

501 Probe element. With regard to the probe face, while group differences in the HR responsivity

502 were not apparent, our exploratory analyses did identify a reduction in SCR. The probe face

503 element of the task required the participants to make a decision as to whether the face was a

504 match or mismatch. Because alcoholics may suffer from decision-making impairments,

505 Bechara and colleagues employed a gambling task while measuring SCRs during task

506 performance to investigate somatic markers of substance abuse (Bechara et al., 2005a;

507 Bechara \& Damasio, 2002). The somatic-marker hypothesis posits that decision-making is a

508 process that depends on emotion, and that deficits in emotional signaling lead to poor

509 decision-making. The investigators found that alcoholic subjects were impaired on the task

510 and unable to generate anticipatory SCRs while pondering risky choices, and the authors

511 made a connection between compulsive/uncontrollable behavior and drinking. They also

512 noted that a subgroup of substance dependent individuals who performed poorly (opting for

513 high immediate gains in spite of future losses) had impaired anticipatory SCRs linked to

514 dysfunction of the ventromedial prefrontal cortex and the amygdala. However, because a

515 group of nonalcoholic patients with ventromedial prefrontal damage could generate SCRs 
516 when they received a reward or a punishment, while patients with amygdala damage could

517 not, the investigators suggested that the roles played by the amygdala and ventromedial

518 prefrontal cortex in decision-making are different. Therefore, it is possible that our results,

519 confirming our hypothesis that ALC participants would demonstrate abnormal SCRs, could be

520 accounted for by the use of different decision-making processes compared to NC

521 participants.

522 Gender differences. For all three aspects of the task wherein SCR reductions were identified

523 among alcoholics relative to controls, this reduction was particularly pronounced in the

524 women, suggesting a greater sensitivity among women to alcohol's long-term effects on

525 electrodermal responsivity. Regardless of alcoholism status, men showed a larger impact of

526 match status on SCR responses to the probe element, with men having higher SCR

527 responses when the probe face matched than when it was a mismatch (Figure 5).

528 Gender differences in HR responses were most evident following the encoded faces,

529 with alcoholic women showing stronger HR response reductions than control women. The

530 general trend toward increased HR responsivity to the distractors among alcoholics was

531 clearest in the women. By contrast, the dampened HR response to the probe faces among

532 alcoholics was similar for both men and women.

533 The tendency for women to display greater effects of alcoholism on ANS responses to

534 the task element may be reflective of gender differences typically reported in

535 psychophysiological responses to emotional stimuli (e.g., Bianchin \& Angrilli, 2012). Gender

536 in relation to peripheral and central nervous system effects of alcoholism only recently has

537 been the focus of intensive research, and additional studies are needed (Ammendola et al.,

538 2000; Devaud, Alele, \& Ritu, 2003; Ruiz \& Oscar-Berman, 2013). 


\section{Conclusions}

541 The pattern of psychophysiological reactions we observed was abnormal for the ALC group,

542 depending upon the type of distractor and facial expression presented. For SCR, the ALC

543 group had consistently reduced responsivity regardless of the task element. For HR, the

544 effect of alcoholism was dependent upon the elements of the task involved. The pattern of

545 ANS activity to emotionally laden stimuli is complex, involving widespread brain circuitry. For

546 both SCR and HR, the alcoholism-related findings were likely due to abnormalities in the

547 mesocorticolimbic system that controls affective functioning (Oscar-Berman et al., 2014). Our

548 results also supported the notion of gender differences in association with long-term chronic

549 alcoholism. Men and women differed with respect to ANS responses, with the alcoholic

550 women generally showing more clear-cut effects than their male counterparts.

552 Acknowledgements

553 This project was supported by funds from the Research Service of the US Department of

554 Veterans Affairs, and by the following grants from the National Institute on Alcohol Abuse and

555 Alcoholism, National Institutes of Health: R01AA07112, K05AA00219, K01AA13402,

556 R01AA016624. The project described was also supported by Grant Number 1 UL1

557 RR025758-04, Harvard Clinical and Translational Science Center, from the National Center

558 for Research Resources. Burke Q. Rosen assisted with data processing and statistical

559 analyses. Diane Merritt and Maria Valmas helped with patient recruitment and

560 neuropsychological assessment. 
562

563

564

565

566

567

568

569

570

571

572

573

574

575

576

577

578

579

580

581

582

583

584 Psychophysiology. $3^{\text {rd }}$ Edition. Cambridge University Press, New York, pp. 182-210.

\section{References}

Ammendola, A., Gemini, D., lannaccone, S., Argenzio, F., Ciccone, G., Ammendola, E., Serio, L., Ugolini, G., \& Bravaccio, F. (2000). Gender and peripheral neuropathy in chronic alcoholism: A clinical-electroneurographic study. Alcohol and Alcoholism, 35(4), 368-371.

Andreassi, J. L. (2007). Psychophysiology. Human Behavior and Physiological Response. $5^{\text {th }}$ Edition. Lawrence Erlbaum Associates, Mahwah, NJ.

APA (2000). Diagnostic and Statistical Manual of Mental Disorders, DSM-IV-TR. American Psychiatric Association, Washington, D.C.

Bechara, A. \& Damasio, H. (2002). Decision-making and addiction (part I): impaired activation of somatic states in substance dependent individuals when pondering decisions with negative future consequences. Neuropsychologia, 40(10), 1675-1689.

Bechara, A., Damasio, H., Damasio, A.R., \& Lee, G.P. (1999). Differential contributions of the human amygdala and ventromedial prefrontal cortex to decision making. Journal of Neuroscience, 19(13), 5473-5481.

Bechara, A., Damasio, H., Tranel, D., \& Damasio, A.R. (2005a). The lowa Gambling Task and the somatic marker hypothesis: some questions and answers. Trends in Cognitive Science, 9, 159-164.

Bechara, A., Tranel, D., Damasio, H., \& Damasio, A.R. (2005b). Failure to respond autonomically to anticipated future outcomes following damage to prefrontal cortex. Cerebral Cortex, 6(2), 215-225.

Berntson, G.G., Quigley, K.S., \& Lozano, D., (2007). Cardiovascular psychophysiology. In:

83 J.T. Cacioppo, L.G. Tassinary, \& G.G. Berntson, eds. The Handbook of 
585 Bianchin, M. \& Angrilli, A. (2012). Gender differences in emotional responses: A

586 psychophysiological study. Physiology \& Behavior, 105(4), 925-932.

587 Boschloo, L., Vogelzangs, N., Licht, C.M.M., Vreeburg, S.A., Smit, J.H., van den Brink, W.,

588 Veltman, D.J., de Geus, E.J.C., Beekman A.T.F., \& Penninx, B.W.H.J. (2011). Heavy

589 alcohol use, rather than alcohol dependence, is associated with dysregulation of the

590 hypothalamic-pituitary-adrenal axis and the autonomic nervous system. Drug and Alcohol

591 Dependence, 116(1-3), 170-176.

592 Boucsein, W. (2012). Electrodermal Activity. Springer, New York.

593 Cahalan, D., Cisin, I., \& Crossley, H. (1969). American Drinking Practices: A National Study

594 of Drinking Behavior and Attitudes (Monograph No. 6). Rutgers Center for Alcohol

$595 \quad$ Studies, New Brunswick, NJ.

596 Carter, B.L. \& Tiffany, S.T. (1999). Meta-analysis of cue-reactivity in addiction research.

$597 \quad$ Addiction, 94, 327-340.

598 Clore, G.L. \& Ortony, A. (2000). Cognition in emotion: Always, sometimes, or never? In:

599 Cognitive Neuroscience of Emotion. Vol., R.D. Lane, L. Nadel, eds. Oxford University

$600 \quad$ Press, New York, pp. 24-61.

601 Cooney, N.L., Litt, M.D., Morse, P.A., Bauer, L.O., \& Gaupp, L. (1997). Alcohol cue reactivity, 602 negative-mood reactivity, and relapse in treated alcoholic men. Journal of Abnormal

603 Psychology, 106(2), 243-250.

604 Critchley, H.D. (2002). Electrodermal responses: What happens in the brain? The $605 \quad$ Neuroscientist, 8, 132-142.

606 Critchley, H. D. (2005). Neural mechanisms of autonomic, affective, and cognitive integration.

607 The Journal of Comparative Neurology, 493(1), 154-166. 
608 Critchley, H.D., Eccles, J., \& Garfinkel, S.N. (2013). Interaction between cognition, emotion, 609 and the autonomic nervous system. In R.M. Buijs \& D.F. Swaab, eds. Handbook of 610 Clinical Neurology, Volume 117, Autonomic Nervous System. Elsevier, Edinburgh, pp. 5961177.

612 Croissant, B., Rist, F., Demmel, R., \& Olbrich, R. (2006). Alcohol-induced heart rate response 613 dampening during aversive and rewarding stress paradigms in subjects at risk for 614 alcoholism. International Journal of Psychophysiology, 61(2), 253-261.

615 Damasio, A.R. (1998). Emotion in the perspective of an integrated nervous system. Brain Research Reviews, 26, 83-86.

617 Davidson, R.J., Jackson, D.C., \& Kalin, N.H. (2000). Emotion, plasticity, context, and 618 regulation: Perspectives from affective neuroscience. Psychological Bulletin, 126, 890619909.

620 Dawson, M.E., Schell, A.M., Filion, D.L., 2007. The electrodermal system. In: Handbook of 621 Psychophysiology. 3rd Edition. J.T. Cacioppo, L.G. Tassinary, \& G.G. Berntson, eds. 622 Cambridge University Press, New York, pp. 159-181.

623 Devaud, L.L., Alele, P., \& Ritu, C. (2003). Sex differences in the central nervous system 624 actions of ethanol. Critical Reviews in Neurobiology, 15(1), 41-59.

625 Dolcos, F. \& McCarthy, G. (2006). Brain systems mediating cognitive interference by 626 emotional distraction. Journal of Neuroscience, 26(7), 2072-2079.

627 Finn PR, Justus AN, Mazas C, Rorick L, Steinmetz JE (2001) Constraint, alcoholism, and 628 electrodermal response in aversive classical conditioning and mismatch novelty 629 paradigms. Integr Physiol Behav Sci 36(2):154-67.

630 Foisy, Marie-Line, Charles Kornreich, Anaïs Fobe, Laetitia D'Hondt, Isidore Pelc, Catherine 631 Hanak, Paul Verbanck, and Pierre Philippot. 2007. "Impaired Emotional Facial Expression 
Recognition in Alcohol Dependence: Do These Deficits Persist With Midterm Abstinence?" Alcoholism, Clinical and Experimental Research 31 (3). John Wiley \& Sons: 404-10.

634 Fowles, D.C., 1993. Electrodermal activity and antisocial behavior: Empirical findings and 635 theoretical issues. In: Progress in electrodermal research. NATO ASI Series A, Vol. 249. J.-C. Roy, W. Boucsein, D.C. Fowles, J.H. Gruzelier, eds. Plenum Press, London, pp. 223-237.

Gainotti, G. (2000). Neuropsychological theories of emotion. In The Neuropsychology of Emotion, Borod, J. C. (Ed.), Oxford, New York, pp. 214-236.

Grüsser, S.M., Wrase, J., Klein, S., Hermann, D., Smolka, M.N., Ruf, M., Weber-Fahr, W., Flor, H., Mann, K., Braus, D.F., \& Heinz, A. (2004). Cue-induced activation of the striatum and medial prefrontal cortex is associated with subsequent relapse in abstinent alcoholics. Psychopharmacology (Berlin), 175(3), 296-302.

Hamilton, M.A. (1960). A rating scale for depression. Journal of Neurology, Neurosurgery, and Psychiatry, 23, 56-62.

Hariri, A.R., Mattay, V.S., Tessitore, A., Fera, F., \& Weinberger, D.R. (2003). Neocortical modulation of the amygdala response to fearful stimuli. Biological Psychiatry, 53, 494-501.

Karpyak, V.M., Romanowicz, M., Schmidt, J.E., Lewis, K.A., \& Bostwick, J.M. (2014).

Characteristics of heart rate variability in alcohol-dependent subjects and nondependent chronic alcohol users. Alcoholism: Clinical and Experimental Research, 38(1), 9-26.

655 affective, facial, visceral, and behavioral reactions. Psychophysiology, 30, 261-73. 
656 Low, P.A., Walsh, J.C., Huang, C.Y., \& McLeod, J.G. (1975). The sympathetic nervous 657 system in alcoholic neuropathy. A clinical and pathological study. Brain. 98, 357-64.

658 MacLaren, V.V. (2001). A quantitative review of the guilty knowledge test. Journal of Applied 659 Psychology, 86, 674-83.

660 Makris, N., Oscar-Berman, M., Jaffin, S.K., Hodge, S.M., Kennedy, D.N., Caviness, V.S., 661 Marinkovic, K., Breiter, H.C., Gasic, G.P., \& Harris, G.J. (2008). Decreased volume of the 662 brain reward system in alcoholism. Biological Psychiatry, 64(3), 192-202.

663 Maltzman, I. (1990). The OR and significance. Pavlovian Journal of Biological Science, 25, $664 \quad 111-121$.

665 Maltzman I, Marinkovic K (1996) Alcohol, alcoholism, and the autonomic nervous system: A 666 critical account, in The Pharmacology of Alcohol and Alcohol Dependence, (Begleiter H, 667 Kissin B Eds), pp 248-306. Oxford University Press, New York.

668 Mangina, C.A. \& Beuzeron-Mangina, J.H. (1996). Direct electrical stimulation of specific 669 human brain structures and bilateral electrodermal activity. International Journal of 670 Psychophysiology, 22, 1-8.

671 Marinkovic, K., \& Halgren, E. (1998). Human brain potentials related to the emotional 672 expression, repetition, and gender of faces. Psychobiology, 26(4), 348-356.

673 Marinkovic, K., Halgren, E., \& Maltzman, I. (2001). Arousal-related P3a to novel auditory 674 stimuli is abolished by moderately low alcohol dose. Alcohol and Alcoholism, 36, 529-539.

675 Marinkovic, K., Oscar-Berman, M., Urban, T., O’Reilly, C.E., Howard, J.A., Sawyer, K., \& 676 Harris, G.J. (2009). Alcoholism and dampened temporal limbic activation to emotional 677 faces. Alcoholism: Clinical and Experimental Research, 33(11), 1880-1892. 
678 Myrick, H., Anton, R.F., Li, X., Henderson, S., Drobes, D., Voronin, K., \& George, M.S. (2004).

679 Differential brain activity in alcoholics and social drinkers to alcohol cues: relationship to 680 craving. Neuropsychopharmacology, 29, 393-402.

681 Naqvi, N.H., \& Bechara, A. (2006). Skin conductance: A psychophysiological approach to the 682 study of decision making. In: Methods in mind. C. Senior, T. Russell, M. Gazzaniga, eds. 683 MIT Press, Cambridge, MA, pp. 103-122.

684 Nesic, J. \& Duka, T. (2006). Gender specific effects of a mild stressor on alcohol cue 685 reactivity in heavy social drinkers. Pharmacology, Biochemistry, and Behavior, 83, 239686248.

687 Novak, D.J., \& Victor, M. (1974). The vagus and sympathetic nerves in alcoholic 688 polyneuropathy. Archives of Neurology, 30, 273-84.

689 O’Daly, O.G., Trick, L., Scaife, J., et al. (2012). Withdrawal-associated increases and 690 decreases in functional neural connectivity associated with altered emotional regulation in 691 alcoholism. Neuropsychopharmacology, 37(10), 2267-2276.

692 Oscar-Berman, M. \& Gade, A. (1979). Electrodermal measures of arousal in humans with 693 cortical or subcortical brain damage. In H.D. Kimmel, E.H. van Olst, \& J.F. Orlebeke 694 (Eds.), The Orienting Reflex in Humans. Lawrence Erlbaum Associates, Hillsdale, NJ, pp. $695 \quad 665-676$.

696 Oscar-Berman, M., Valmas, M., Sawyer, K.S., Ruiz, S.M.M., Luhar, R., \& Gravitz, Z. (2014). 697 Profiles of impaired, spared, and recovered neuropsychological processes in alcoholism. 698 In Pfefferbaum, A. \& Sullivan, E.V. (Eds.), Handbook of Clinical Neurology: Alcohol and 699 the Nervous System. Elsevier: Edinburgh (2014). 
700 Pomerleau OF, Fertig J, Baker L, Cooney N (1983) Reactivity to alcohol cues in alcoholics 701 and non-alcoholics: implications for a stimulus control analysis of drinking. Addict Behav $7028(1): 1-10$.

703 Ray, L. A., McGeary, J., Marshall, E., \& Hutchison, K. E. (2006). Risk factors for alcohol 704 misuse: examining heart rate reactivity to alcohol, alcohol sensitivity, and personality 705 constructs. Addictive Behaviors, 31(11), 1959-1973.

706 Reid MS, Flammino F, Starosta A, Palamar J, Franck J (2006) Physiological and subjective 707 responding to alcohol cue exposure in alcoholics and control subjects: evidence for 708 appetitive responding. J Neural Transm 113(10):1519-35.

709 Robins, L., Cottler, L., Bucholz, K., Compton, W., North, C., \& Rourke, K. (2000). Diagnostic 710 Interview Schedule for the DSM-IV. Washington University School of Medicine, St. Louis, 711 MO.

712 Roy, M., Shohamy, D., \& Wager, T.D. (2012). Ventromedial prefrontal-subcortical systems 713 and the generation of affective meaning. Trends in Cognitive Science, 16(3): 147-156.

714 Ruiz, S.M.M. (2012). Gender differences in brain structure and function in alcoholism. Ph.D.

715 Dissertation, Boston University. ProQuest, UMI Dissertations Publishing, 3500670.

716 Ruiz, S.M.M. \& Oscar-Berman, M. (2013). Closing the gender gap: The case for gender717 specific alcoholism research. Journal of Alcoholism \& Drug Dependence, 1(6), 1-3.

718 Salloum, J.B., Ramchandani, V.A., Boduka,.J., et al. (2007). Blunted rostral anterior cingulate 719 response during a simplified decoding task of negative emotional facial expressions in 720 alcoholic patients. Alcoholism Clinical and Experimental Research, 31(9),1490-1504.

721 Schell, A.M., Dawson, M.E., Rissling, A., Ventura, J., Subotnik, K.L., Gitlin, M.J., \& 722 Nuechterlein, K.H. (2005). Electrodermal predictors of functional outcome and negative 723 symptoms in schizophrenia. Psychophysiology, 42, 483-92. 
724 Sinha, R., Fox, H.C., Hong, K.A., Bergquist, K., Bhagwagar, Z., \& Siedlarz, K.M. (2009).

725 Enhanced negative emotion and alcohol craving, and altered physiological responses

726 following stress and cue exposure in alcohol dependent individuals.

$727 \quad$ Neuropsychopharmacology, 34, 1198-1208.

728 Staiger, P K, and J M White. 1991. "Cue Reactivity in Alcohol Abusers: Stimulus Specificity

729 and Extinction of the Responses." Addictive Behaviors 16 (5): 211-21.

730 Starcke, K., van Holst, R.J., van den Brink, W., Veltman, D.J., \& Goudriaan, A.E. (2013).

731 Physiological and endocrine reactions to psychosocial stress in alcohol use disorders:

732 Duration of abstinence matters. Alcoholism: Clinical and Experimental Research, 37(8),

$733 \quad 1343-1350$.

734 Stormark, K.M., Laberg, J.C., Nordby, H., \& Hugdahl, K. (2000). Alcoholics' selective attention

735 to alcohol stimuli: automated processing? Journal of Studies on Alcohol, 61(1), 18-23.

736 Stritzke, W.G.K., Breiner, M.J., Curtin, J.J., \& Lang, A.R. (2004). Assessment of substance

737 cue reactivity: Advances in reliability, specificity, and validity. Psychology of Addictive

738 Behaviors, 18(2), 148-159.

739 Stuss, D., \& Knight, R.T., Eds. (2012). Principles of frontal lobe function. Oxford University $740 \quad$ Press, New York.

741 Szegedi, A., Lörch, B., Scheurich, A., Ruppe, A., Hautzinger, M. \& Wetzel, H. (2000). Cue 742 exposure in alcohol dependent patients: preliminary evidence for different types of cue 743 reactivity. Journal of Neural Transmission, 107(6), 721-730.

744 Thorell, L.H. (2009). Valid electrodermal hyporeactivity for depressive suicidal propensity 745 offers links to cognitive theory. Acta Psychiatrica Scandinavica, 119, 338-49. 
746 Tranel, D. (2000). Electrodermal activity in cognitive neuroscience: Neuroanatomical and 747 neuropsychological correlates. In: Cognitive Neuroscience of Emotion. R.D. Lane, L.

$748 \quad$ Nadel, eds. Oxford University Press, New York, pp. 192-224.

749 Wechsler, D. (1997). WAIS-III and WSM-III Technical Manual. Harcourt Brace \& Company, 750 San Antonio.

751 Weisbard, C, and P K Graham. 1971. "Heart-Rate Change as a Component of the Orienting 752 Response in Monkeys." Journal of Comparative and Physiological Psychology 76 (1): 7475383.

754 Woodward, J.A., Bonett, D.G., \& Brecht, M.L. (1990). Introduction to linear models and 755 experimental design. Harcourt Brace Jovanovich, San Diego.

756 Wrase, J., Grüsser, S.M., Klein, S., Diener, C., Hermann, D., Flor, H., Mann, K., Braus, D.F. \& 757 Heinz, A. (2002). Development of alcohol-associated cues and cue-induced brain 758 activation in alcoholics. European Psychiatry, 17(5), 287-291. 


\section{Table $\mathbf{1}_{\text {(on next page) }}$}

Characteristics of the research participants.

Means and standard deviations (SD) are displayed for age, education, measures of drinking history, the Wechsler Adult Intelligence Scale (WAIS) and the Wechsler Memory Scale (WMS). Besides amount and duration of drinking, these characteristics did not differ significantly between the ALC and NC groups, with the exception of education, for which the NC group had more years on average $(95 \% \mathrm{Cl}:[0.0,1.7]$ years). Supplemental Tables 1 and 2 describe the subgroups examined for heart rate and SCR. For those samples, the ALC and NC groups had similar education levels. ${ }^{1}$ One man drank during his time in the military service for about 2.5 years, decades prior to the scan, but this drinking was not severe and he reported that it did not impact his occupation, health, or personal life. Another man reported drinking heavily for 0 years in a prior study in our lab, but then after answering the same questionnaire for the present study, he reported that he recalled that, decades prior to the scan, he drank over 21 drinks per week for approximately a year. One woman reported that at most she drank a half bottle of wine a day ( 1 to 3 drinks) for at most 5 years, so we used 5 years as a conservative estimate. In prior (and subsequent) studies in our laboratory, she reported drinking less, so her DHD was set to 0 for those studies. All three participants had not been drinking heavily recently. 
Age (years)

Education (years)

Duration of Heavy Drinking (years)

Daily Drinks

Length of Sobriety (years)

WAIS-III Full Scale IQ

WAIS-III Verbal IQ

WAIS-III Performance IQ

WMS-III Immediate Memory

WMS-III Delayed Memory

WMS-III Working Memory
Age (years)

Education (years)

Duration of Heavy Drinking (years) ${ }^{1}$

Daily Drinks

Length of Sobriety (years)

WAIS-III Full Scale IQ

WAIS-III Verbal IQ

WAIS-III Performance IQ

WMS-III Immediate Memory

WMS-III Delayed Memory

WMS-III Working Memory
Alcoholic Participants

All $(\mathrm{N}=32) \quad$ Women $(\mathrm{N}=16) \quad$ Men $(\mathrm{N}=16)$

Mean SD

$55.2 \quad 9.9$

$14.9 \quad 2.0$

$16.2 \quad 5.9$

$11.7 \quad 9.3$

$8.8 \quad 11.2$

$109.1 \quad 14.3$

$111.5 \quad 12.0$

$104.1 \quad 17.4$

$109.8 \quad 17.9$

$112.0 \quad 18.5$

$103.6 \quad 12.2$

Mean

SD

Mean

SD

$\begin{array}{llll}56.9 & 9.4 & 53.5 & 10.5\end{array}$

$\begin{array}{llll}15.7 & 2.0 & 14.1 & 1.8\end{array}$

$\begin{array}{llll}15.3 & 4.9 & 17.1 & 6.9\end{array}$

$\begin{array}{llll}10.1 & 8.3 & 13.4 & 10.3\end{array}$

$\begin{array}{llll}11.5 & 12.0 & 5.9 & 9.8\end{array}$

$\begin{array}{llll}110.1 & 13.0 & 108.1 & 15.8\end{array}$

$\begin{array}{llll}112.8 & 11.6 & 110.3 & 12.6\end{array}$

$\begin{array}{llll}104.9 & 16.4 & 103.3 & 18.8\end{array}$

$\begin{array}{llll}113.6 & 20.4 & 105.9 & 14.7\end{array}$

$\begin{array}{llll}115.6 & 22.9 & 108.4 & 12.3\end{array}$

$\begin{array}{llll}104.8 & 12.4 & 102.4 & 12.4\end{array}$

Nonalcoholic Participants

\begin{tabular}{cccccc}
\multicolumn{2}{c}{ All $(\mathbf{N}=\mathbf{3 0})$} & \multicolumn{2}{c}{ Women $(\mathbf{N}=15)$} & \multicolumn{2}{c}{ Men $(\mathbf{N}=\mathbf{1 5})$} \\
Mean & SD & Mean & SD & Mean & SD \\
52.6 & 12.8 & 54.7 & 15.2 & 50.5 & 10.0 \\
15.9 & 2.1 & 16.1 & 2.4 & 15.6 & 1.8 \\
0.1 & 0.5 & 0.0 & 0.0 & 0.3 & 0.7 \\
0.3 & 0.6 & 0.3 & 0.7 & 0.3 & 0.5 \\
NA & NA & NA & NA & NA & NA \\
111.5 & 13.9 & 114 & 17.2 & 109.2 & 10.0 \\
113.3 & 15.0 & 114.2 & 17.7 & 112.5 & 12.5 \\
107.4 & 14.5 & 110.8 & 14.5 & 104.3 & 14.2 \\
111.1 & 16.3 & 115.2 & 17.2 & 107.2 & 14.9 \\
110.5 & 14.1 & 112.2 & 14.5 & 108.9 & 14.1 \\
106.3 & 12.7 & 108.6 & 13.5 & 104.2 & 11.9
\end{tabular}




\section{1}

This figure shows the flow of the delayed match-to-sample task paradigm.

Rows provide examples of the three facial emotion types (positive, neutral, negative) and the three distractor types (scrambled, nonalcoholic, alcoholic). The rows also represent both matching and non-matching probe faces (match, nonmatch, match). The faces have been obscured in this publication to protect the identity of the individuals who were photographed. The participants saw the entire un-obscured faces.

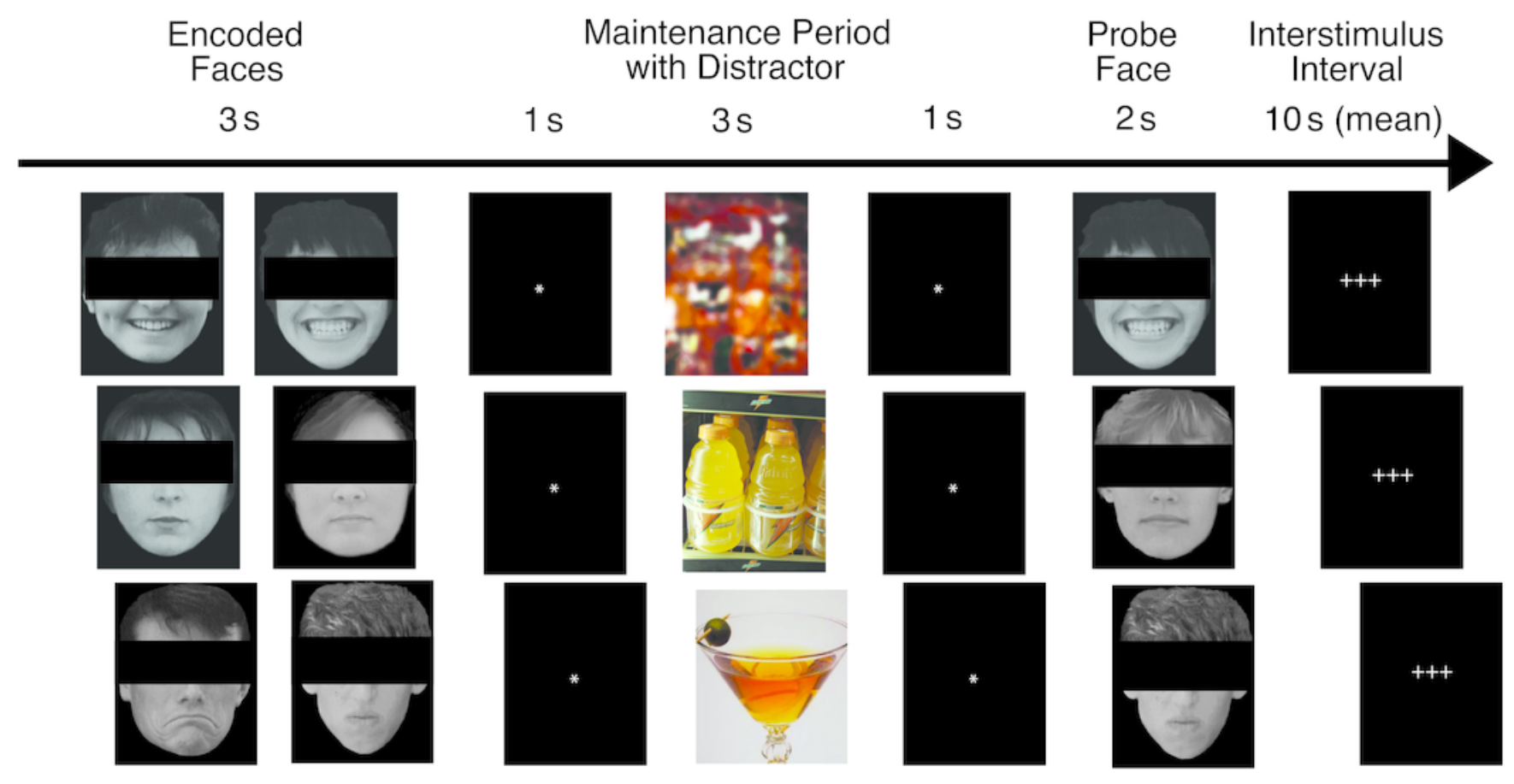


2

For the encoded faces, alcoholic participants had fewer skin conductance responses (SCR) than nonalcoholic participants.

Data are presented for alcoholic participants (ALC, in red) and nonalcoholic participants (NC, in blue), split by gender. The asterisk indicates a significant difference, and error bars represent standard error. No significant interactions were identified.

(A) ALC women had significantly fewer SCRs $(>0.03$ microsiemens $(\mu \mathrm{S}))$ to the encoded faces than ALC men.

(B) SCR amplitudes $(\mu \mathrm{S})$ to the same stimuli appeared to be similar.

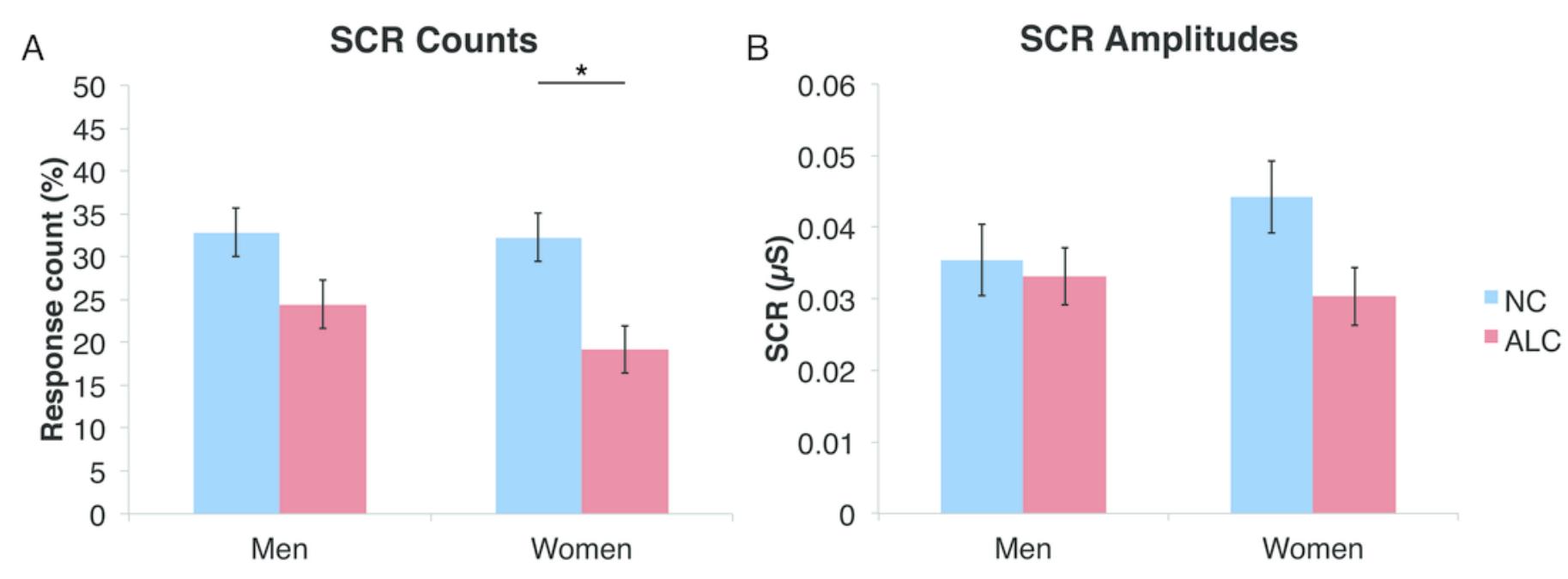




\section{3}

Skin conductance responses (SCR) to distractor cues were reduced for alcoholic participants compared to nonalcoholic controls.

Data are presented for alcoholic participants (ALC, in red) and nonalcoholic participants (NC, in blue), split by gender. Asterisks indicate significant differences, and error bars represent standard error. No significant interactions were identified.

(A) ALC women had significantly fewer SCRs ( $>0.03$ microsiemens $(\mu \mathrm{S})$ ) to the distractor images than ALC men.

(B) SCR amplitudes $(\mu \mathrm{S})$ to the same stimuli revealed a similar pattern.

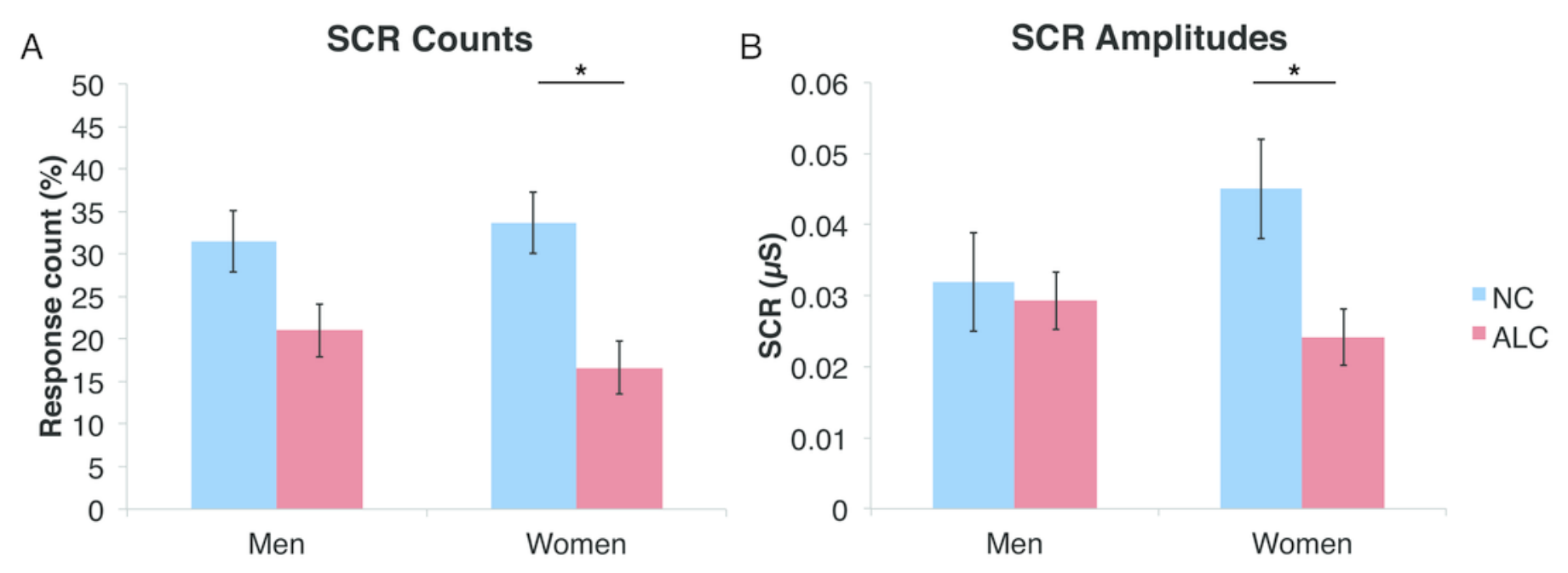


4

Skin conductance responses (SCR) to the distractor cues were not significantly related to the type of distractor for alcoholic participants compared

Data are presented for alcoholic participants (ALC, in red) and nonalcoholic participants (NC, in blue). Error bars represent standard error.

(A) A significant interaction of Group x Distractor Type was not identified for SCR counts.

(B) SCR amplitudes ( $\mu \mathrm{S})$ also did not reveal a significant interaction.

A

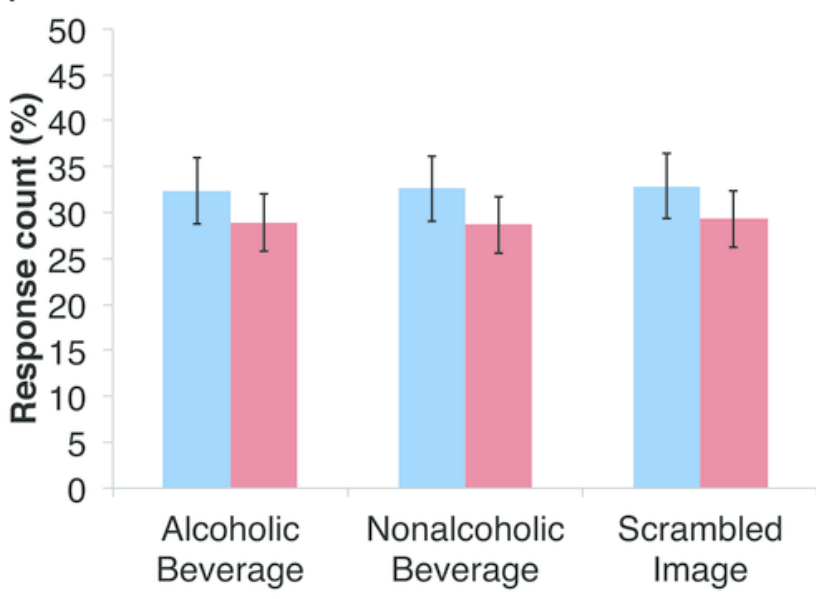

B

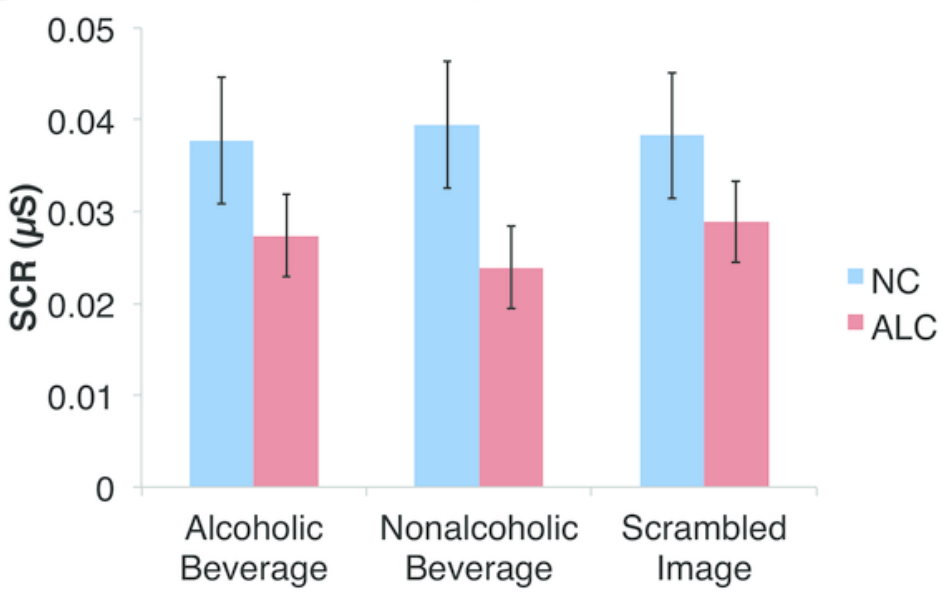




\section{5}

Skin conductance responses (SCR) to probe faces were reduced for alcoholic participants compared to nonalcoholic controls.

Nonmatch SCRs are in blue and match SCRs are in red. Asterisks indicate significant differences between match status, and error bars represent standard error. No significant interactions were identified.

(A) Compared to women, men had significantly more SCRs ( $>0.03$ microsiemens $(\mu \mathrm{S}))$ to the matching probe faces than the nonmatching probe faces. The data are shown for all alcoholic and nonalcoholic participants together.

(B) For neutral and positive faces, SCR amplitudes were higher for faces that matched the encoded faces. Data are shown for alcoholic and nonalcoholic men and women together.

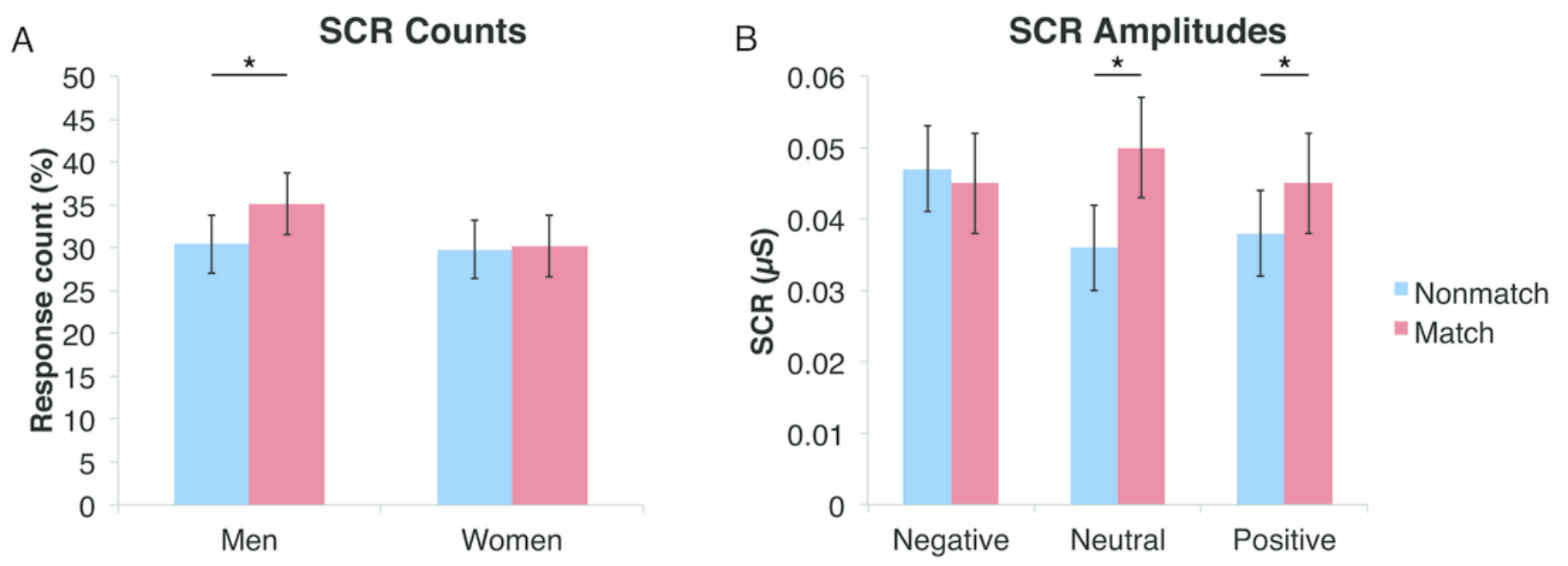




\section{6}

Heart rate (HR) tended to decline more in alcoholics in response to the encoded faces than in nonalcoholics.

Bar height indicates beats per minute (BPM), with standard error bars. Data are presented for alcoholic participants (ALC, in red) and nonalcoholic participants (NC, in blue), split by gender. The asterisk indicates a significant difference, and error bars represent standard error. No significant interactions were identified.

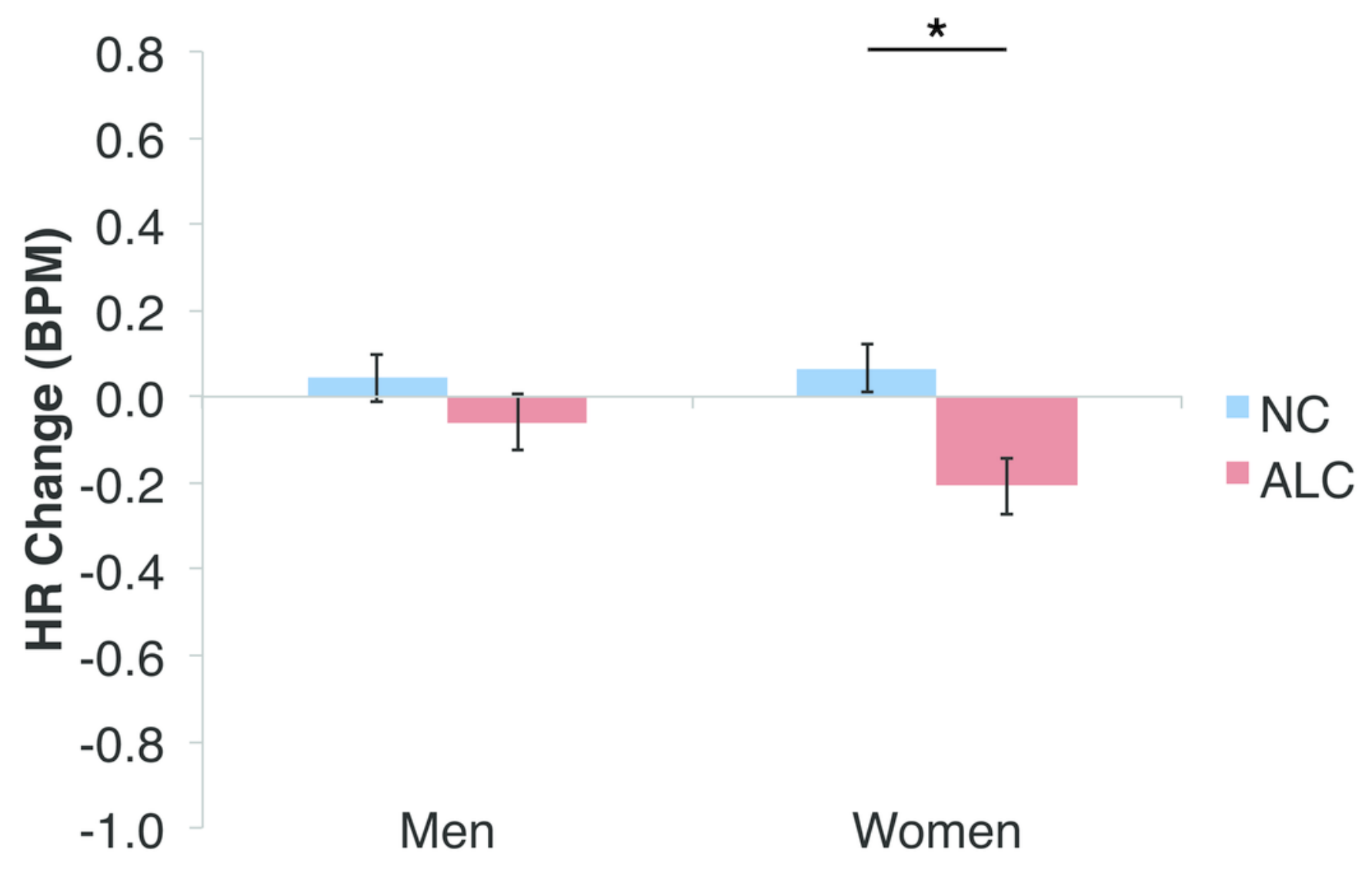




\section{7}

The increase in heart rate (HR) to distractor cues depended upon the group and the emotion of the preceding face.

Heart rate (HR) increased by 0.3 beats per minute (BPM) in response to the distractor element, and a significant Group x Gender x Face Valence interaction was identified. Data are presented for alcoholic participants (ALC, in red) and nonalcoholic participants (NC, in blue). Error bars represent standard error.
(A) Shows values for men.
(B) Shows values for women.

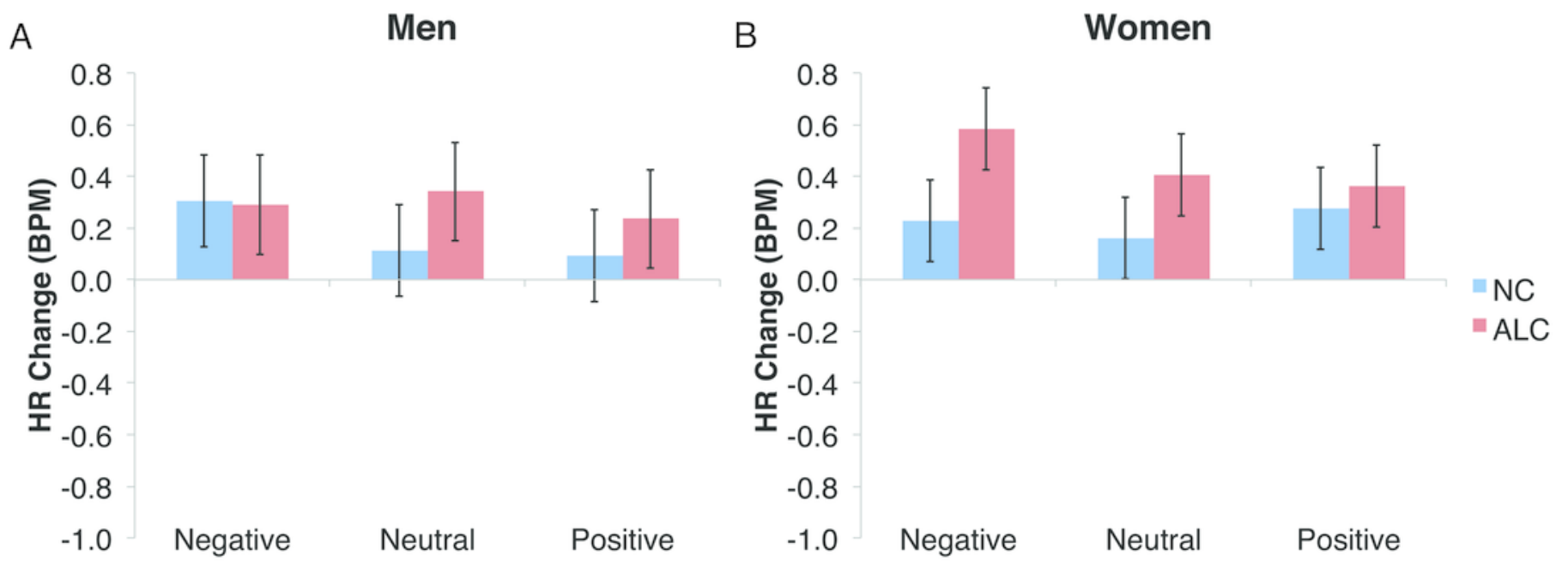


8

Heart Rate (HR) responses to the distractor cues were not significantly related to the type of distractor for alcoholic participants compared to nona

Data are presented for alcoholic participants (ALC, in red) and nonalcoholic participants (NC, in blue). Error bars represent standard error. A significant interaction of Group x Distractor Type was not identified for HR counts.

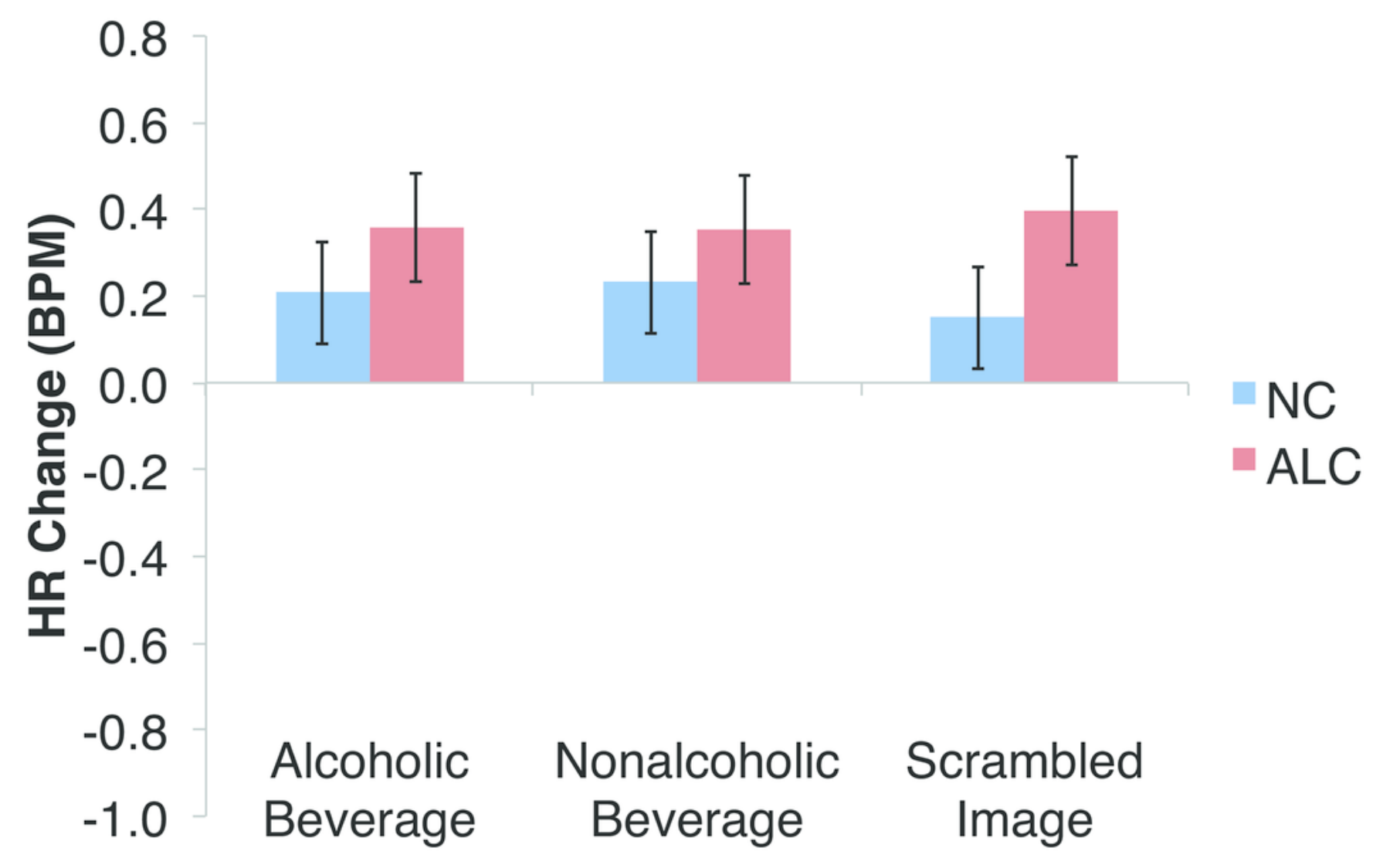


9

For the probe face, heart rate (HR) significantly declined but was not significantly related to the group, the type of distractor, or the emotion of

Bar height indicates beats per minute (BPM), with standard error bars. Data are presented for alcoholic participants (ALC, in red) and nonalcoholic participants (NC, in blue), split by gender. No significant interactions were identified.

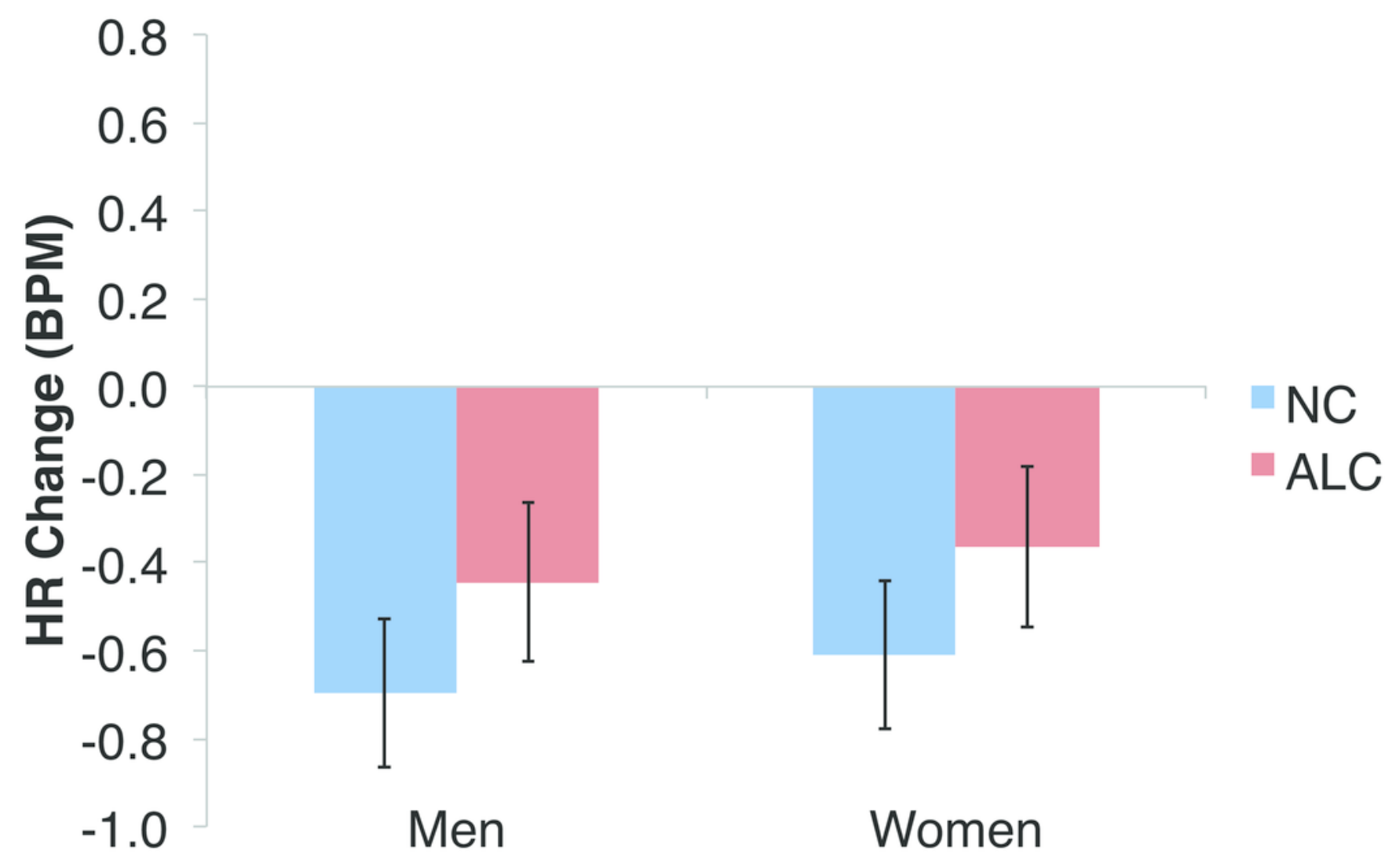

